\title{
Method of thermometric determination of thermophysical characteristics of thermolabs materials
}

\author{
Olga Mazurenko, Liliia Kharchenko, \\ Dmytro Kolomiets, Oleksandr Mazurenko
}

\section{National University of food Technologies, Kyiv, Ukraine}

Keywords:

Thermometric

Heat transfer

Metrology

Determination

Thermolabs

\section{Article history:}

Received 12.07.2018

Received in revised form

21.12.2018

Accepted 28.03.2019

\section{Corresponding author:}

Dmytro Kolomiets

E-mail:

kdp1210@i.ua

DOI: $10.24263 / 2304$

974X-2019-8-1-14

\section{Abstract}

Introduction. The analytical researches of methods and devices of determination of thermophysical properties of complicated structure and chemical composition of products of processing industry are carried out in order to increase the accuracy of measurements.

Materials and methods. The basis of the studied devices is thermoelectric converters of temperature and heat flow in the form of "auxiliary wall". To improve the accuracy of the measurements of the thermophysical characteristics of materials, the graph-analytical method of calibration of the devices was used.

Results and discussion. To study the thermophysical characteristics of thermolabial materials, the most suitable thermometric means of their complex definition, which allow to conduct research in the presence or absence of material phase transformations of its constituents. The calorimetric measuring instruments are based on the measurement of the temperature of the heat fluxes that penetrate the sample under different thermal conditions.

Thermal, electric and other transmission processes in thermometric materials determine the instability of the transformation function and form an instrumental error. On the basis of the calculation and graphic analysis of the thermal and capacitive resistance of the "device-sampler" system, a fundamentally new method has been developed, according to which the thermophysical characteristics of the material and the metrological characteristics of the device are determined in a complex and simultaneously based on the results of the experiment with samples of only the test material.

Conclusion. Simultaneous obtaining information on the values of the thermophysical characteristics of the material and the metrological characteristics of the device can improve the accuracy of the determination of the thermophysical characteristics of products. 


\section{Introduction}

The intensity of heat exchange processes occurring in substances (materials), is quantified by changing their thermophysical characteristics (hereinafter TPC), in particular the thermal conductivity $\lambda_{x}$, mass $c_{x}$ and volumetric $(c \rho)_{\mathrm{x}}$ heat capacity, temperature diffusivity $a_{x}=\lambda_{x} /(c \rho)_{x}$ and thermal activity $\beta_{x}=\sqrt{\lambda_{x} \cdot(c \rho)_{x}}$. These TPC refer to one of the defining parameters of materials in their manufacturing, storage, application and operation in various industries. Therefore, when calculating both natural thermal processes and processes of heat treatment of materials [1,2], it is necessary to take into account the TPC of those materials, using their known values given in reference literature, for example [3-6].

The accuracy of determination of TPC of materials depends on many factors, ranging from the perfection of methods and devices for determining TPC, primary and secondary means of measurement, and ending with equipment that provides the creation and maintenance of the desired thermal mode in the sample of the research material [7]. In many cases, special methods and devices [7-16] are used to determine the TPC of materials, which can be divided into three groups: determination of thermal conductivity; mass or volumetric heat capacity; and complex determination of TPC of materials - this is when $\lambda_{x}, c_{x}$ or $(c \rho)_{\mathrm{x}}$, as well as $a_{x}$ and $\beta_{x}$ of the material sample, and if necessary, the dependence of these characteristics on temperature are determined simultaneously during one experiment.

We should note that regardless of which of the selected groups includes the selected method and device for measuring the TPC of material, in the process of research it is necessary to take measures to determine the metrological characteristics (hereinafter-MC) of the device, in particular the sensitivity of the primary converters, the resistances of the heat transfer device to the sample of the research material, as well as the amount of heat required to change the temperature of the device, and others.

At the same time, one of the main MC of the device that determines the accuracy of the experimental determination of TPC of materials is ballast thermal $R_{b}$, and capacitive $P_{b}$ resistances. Their value depends on the corresponding resistances of the electrical insulation of the temperature converters (hereinafter TC) and the heat flow converters (hereinafter HFC) of the TPC of device, the resistance of the contact of the sample with the elements of the device, the degree of uniformity of the temperature distribution and the heat flow density on the working surfaces of the device and the sample of the research material, etc. In the general case, to determine these ballast resistances, a series of special experiments with calibration of the device is carried out.

To determine the thermolabile materials TPC, in particular food products, in the temperature range, where phase transformations occur in the sample of the research material, it is advisable to use devices for complex determination of TFC of materials, which allow implementing a combination of stationary and transient modes. In the temperature range at which phase transformations do not occur in the sample of the test product, it is convenient to carry out the determination of TPC of the material in the regular mode of the second kind (quasi-stationary thermal mode) [7].

Given that the errors in determining MC of device to a large extent generally determine the reliability of the measurement results of the TPC of material, the aim of the study is to develop a method for the integrated determination of the MC of device and TPC of thermolabile materials - raw materials, intermediate and finished industrial products, in particular, in the processing industry. 


\section{Materials and methods}

One of the most common devices for determining the thermal conductivity of flat samples of materials in Ukraine $[11,13]$ consists (Figure 1) from a down-flow condenser 1 and an electric heater 2 on the working surfaces of which flat primary heat flow converters 3 and temperature converters 4 are located and between which a sample of the research material 5 is placed.

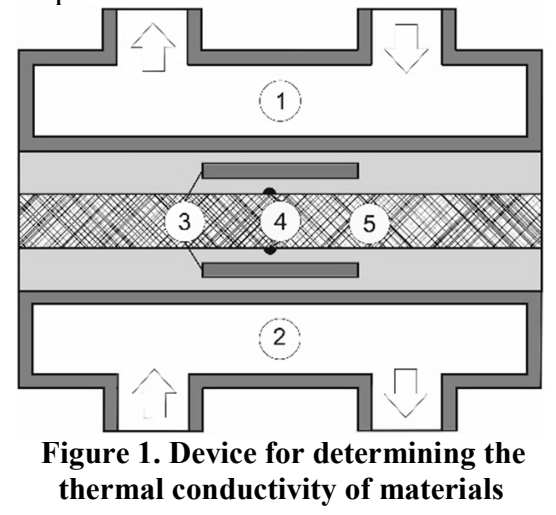

The thermal conductivity of the sample of the research material is calculated from the measurement results in the stationary thermal mode of the temperature difference $\Delta t$ on the surfaces of a flat sample with thickness $h$ and the heat flow density $q$, which passes through the sample, by the formula:

$$
\lambda_{x}=\frac{h}{\Delta t / q-R_{b}},
$$

where $R_{b}$ is an additional (ballast) thermal resistance, the value of which is determined during the calibration of the device.

Similar in structure devices for determining the thermal conductivity of materials are widely used in Germany [14], the United States [15] and other countries.

When determining the heat capacity of materials, so-called conductive microcalorimeters are often used [16]. In these devices, to measure the amount of heat $Q_{m}$, supplied or withdrawn from the device and the sample of the research material with mass $m_{x}$ (volume $V_{x}$ ), which causes a change in the average temperature of the sample by the value $\overline{\delta t}$, the batteries of their thermocouple elements are used. The measuring cell of such devices, where the sample of the research material is located, have the form of a cylindrical thermometric shell or a flat thermoelectric HFC. According to the measurement results, the mass $c_{\mathrm{x}}$ and volumetric $(c \rho)_{\mathrm{x}}$ heat capacity of the material are calculated according to the formulas:

$$
\begin{gathered}
c_{x}=\frac{Q_{m}-Q_{b}}{m_{x} \cdot \bar{\delta}}, \\
(c \rho)_{x}=\frac{Q_{m}-Q_{b}}{V_{x} \cdot \overline{d t}} .
\end{gathered}
$$

where $Q_{b}$ - the amount of heat consumed to change the temperature of the device by $\delta t$. Its value is determined by the calibration of the TPC device.

To date, to determine the heat capacity the methods of heat analysis during linear and step-by-step scanning of the heat capacity by the temperature of the material sample have become widespread [17-21].

Methods for determining the TPC of materials are reflected in the standards of Ukraine, that are fully consistent with the standards of Germany, USA and Japan [22-25].

The devices for complex determination of TPC by the basic structure, in fact, do not differ from the device shown in Figure 1. At the same time, it should be noted that the heat block of these devices can form various heat exchangers [7, 12]. Except for the electric heater and the flow heat exchanger shown in Figure 1, the heat block can be formed from two liquid 
flow heat exchangers; a combination of flow heat exchanger and electric heater; a flow heat exchanger or an electric heater and a device that has a "developed" surface for convective heat withdrawal from the sample of the research material, etc. The application of one or another pair of heat exchangers in a TPC device for complex determination of TPC is mainly determined by the type of thermal modes and the temperature range of the study of TPC of materials [7].

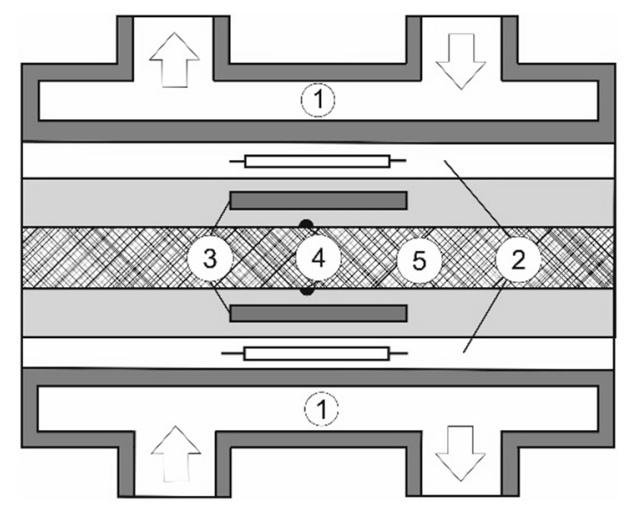

Figure 2. Device for determination of TPC of materials with combined heat block

Under such conditions, for the complex determination of TPC, in particular food products, it is advisable to use a device with a combined heat block (Figure 2). Such a unit consists of two flow heat exchangers 1 and two thin, electric heaters 2 with evenly distributed density of the heat release over the surface, which are located between the heat exchangers and the plate of the measuring cell of the device with the HFC 3 and TC 4 mounted therein. Both flow heat exchangers are supplied with a heat-transfer material of the same temperature that varies linearly over time. In this case, electric heaters are used to create a time-stable difference in the density of the heat flow on the surfaces of the test product 5 .

The thermal conductivity $\lambda_{\mathrm{x}}$ and the volumetric heat capacity $(c \rho)_{\mathrm{x}}$ of the test sample of the product are calculated from the equations obtained as a result of solving the task of unsteady thermal conductivity with bilateral heating of the plate in the regular mode of the second kind [7]. Here, in contrast to the stationary thermal mode, where the temperature of the sample of the research material does not change, when it is heated (cooled) in the quasistationary thermal mode of the flat sample of the research material, the heat flow density on the "working" surfaces of the sample although does not change in time $\left(q_{1}(\tau)=\right.$ const, $q_{2}(\tau)=$ const, $q_{1}(\tau)-q_{2}(\tau)=\Delta q=$ const $)$, but differ in value, that is $q_{1} \neq q_{2}$. At that the temperature on the "working" surfaces of the sample changes linearly: $t_{1}(\tau)-t_{2}(\tau)=\Delta t=$ const $\neq 0$.

Taking into account the ballast thermal $R_{b}$ and capacitive $P_{b}$ resistances of TPC device, the TPC of research material is calculated by the formulas:

$$
\begin{gathered}
\lambda_{x}=\frac{h}{2 \cdot\left(t_{1}-t_{2}\right) /\left(q_{1}+q_{2}\right)-R_{b}}=\frac{h}{(\Delta t / \bar{q})-R_{b}}, \\
(c \rho)_{x}=\frac{\left(q_{1}-q_{2}\right) /(\delta \bar{t} / \Delta \tau)-P_{b}}{h}=\frac{\left(\Delta q / u_{t}\right)-P_{b}}{h}, \\
a_{x}=\frac{\lambda_{x}}{(c \rho)_{x}}=h \cdot\left(\frac{\Delta t}{\bar{q}}-R_{b}\right)^{-1} \cdot\left(\frac{\Delta q}{u_{t}}-P_{b}\right)^{-1} \\
\beta_{x}=\sqrt{\lambda_{x} \cdot(c \rho)_{x}}=\sqrt{\frac{\left(\Delta q / u_{t}\right)-P_{b}}{(\Delta t / \bar{q})-R_{b}}}
\end{gathered}
$$


where $u_{t}=\overline{\delta t} / \Delta \tau=0,5 \cdot\left[\left(t_{1}+t_{2}\right)_{\tau i}-\left(t_{1}+t_{2}\right)_{\tau 0}\right] /\left(\tau_{i}-\tau_{0}\right)-$ the rate of change of the average temperature $\overline{\delta t}$ of the sample of research material in the time interval $\Delta \tau=\tau_{i}-\tau_{0}$ when heating (cooling) of the sample in the quasi-static thermal mode from the average temperature $\bar{t}_{\tau_{0}}=\left(t_{1}+t_{2}\right)_{\tau_{0}} / 2$, at the moment of time $\tau_{0}$, to the average temperature $\bar{t}_{\tau_{i}}=\left(t_{1}+t_{2}\right)_{\tau_{i}} / 2$.

\section{Results and discussion}

From the results of the analysis of formulas (1)-(7) it follows that each of the considered methods of experimental determination of thermal conductivity, volumetric or mass heat capacity, as well as the complex determination of TPC of materials, in fact, is reduced to obtaining an equation or a system of equations of the following form:

$$
P_{m}=P_{x}+P_{b}, \quad R_{m}=R_{x}+R_{b},(8)
$$

where $R_{x}=h / \lambda_{x}$ and $P=h \cdot(c \rho)_{x}$ - respectively, the thermal and volumetric resistances of the sample of the research material; $R_{b}$ and $P_{b}$ - respectively, the ballast thermal and ballast capacitive resistances of the device $R_{m}$ and $P_{\mathrm{m}}$ - respectively, the thermal and volumetric resistances of the "device-sample" system, measured during the experiment on the study of the TPC of the material.

The value of thermal and volumetric resistances of the system "device-sample" $\left(R_{m}, P_{\mathrm{m}}\right)$ is calculated by measuring the values obtained in the course of the experiment on the study of the TPC of the material.

The accuracy of the calculation results of $R_{m}$ and $P_{\mathrm{m}}$ depends on the perfection of the system of maintaining the desired thermal mode in the device (material sample), the device itself, the primary and secondary measuring equipment, as well as the accuracy of determining the operating coefficients $K_{q}$ and $K_{t}$ (inverse sensitivity) of the primary TC and HFC of the device. Therefore, it is natural that the accuracy of the results of the calculations of the value of these resistances directly determines the reliability of the results of determining the TFC of the material.

As it follows from the results of the analysis of the structure of devices, as well as equations (1)-(7) for calculation of TPC of materials, thermal and volumetric resistances of the "device-sample" system are calculated based on the measurement of temperature $\left(t_{1}, t_{2}\right)$ and heat flow density $\left(q_{1}, q_{2}\right)$ on the "working" surfaces of a flat sample of the research material. But in practice, this is somewhat different, since the temperature and heat flow density on the surfaces of the material sample also have to be calculated. Thus, when thermoelectric TC and HFC are used to determine the temperature and density of the heat flow in the TPC device, which generate EMF, respectively, $e_{t 1}, e_{t 2}$ and $e_{q 1}, e_{q 1}$, the calculation equation $R_{m}$ and $R_{m}$ when heating (cooling) the sample in the quasi-stationary thermal mode have the following form:

$$
\begin{gathered}
R_{m}=\frac{\Delta t}{\Sigma q / 2}=\frac{t_{1}-t_{2}}{\left(q_{1}+q_{2}\right) / 2}=\frac{K_{t} \cdot\left(e_{t 1}-e_{e 2}\right)}{K_{q} \cdot\left(e_{q 1}+e_{q 2}\right) / 2} \\
P_{m}=\frac{\Delta q}{u_{t}}=\frac{q_{1}-q_{2}}{\left[\left.\left(t_{1}+t_{2}\right)\right|_{\tau_{i}}-\left.\left(t_{1}+t_{2}\right)\right|_{\tau_{0}}\right] /\left[2 \cdot\left(\tau_{i}-\tau_{0}\right)\right]}=\frac{K_{q} \cdot\left(e_{q 1}-e_{q 2}\right)}{K_{t}\left[\left.\left(e_{t 1}+e_{t 2}\right)\right|_{\tau_{i}}-\left.\left(e_{t 1}+e_{t 2}\right)\right|_{\tau_{0}}\right] /\left[2 \cdot\left(\tau_{i}-\tau_{0}\right)\right]}
\end{gathered}
$$


The operating coefficients $K_{t}$ and $K_{q}$, respectively, TC and TPC or their inverse values - sensitivity, which are used to calculate the temperature $t$ and the heat flow density $q$ on the surfaces of the sample of the research material (differences $\Delta t=t_{1}-t_{2}, \Delta q=q_{1}-q_{2}$, and the average of the values $\left.\bar{t}=\left(t_{1}+t_{1}\right) / 2=\Sigma t / 2, \bar{q}=\left(q_{1}+q_{1}\right) / 2=\Sigma q / 2\right)$ also refer to the MC of the device.

The accuracy of their determination in a series of special experiments with calibration of the device, as well as ballast resistances, significantly affects the reliability of the results of the experimental determination of TPC of materials.

Currently, among the existing methods for determining the MC of TPC devices one of the most common techniques of which is associated with the use of working calibration standards [7-12].

In our opinion, this is due to the fact that the method of determining the MC of device using working calibration standards does not differ from the method of determining the TPC of materials. For example, according to the results of two experiments (further indicated as, respectively, 1 and 2) with samples of standard ( $s$ ) material, which has thermal conductivity $\lambda_{s}$ and volumetric heat capacity $(c \rho)_{s}$, at a thickness of samples $h_{1} \neq h_{2}$, that is, with samples that differ in thermal $R_{s 1} \neq R_{s 2}$ and volumetric $P_{s 1} \neq P_{s 2}$ resistances, we obtain systems composed of equations of the form (7) and (8), respectively :

$$
\begin{gathered}
\left\{\begin{array}{l}
\left(\frac{\Delta t}{\bar{e}_{q}}\right)_{1}=\frac{K_{q}}{\lambda_{s}} \cdot h_{1}+K_{q} \cdot R_{b}=K_{q} \cdot R_{s 1}+K_{q} \cdot R_{b} \\
\left(\frac{\Delta t}{\bar{e}_{q}}\right)_{2}=\frac{K_{q}}{\lambda_{s}} \cdot h_{2}+K_{q} \cdot R_{b}=K_{q} \cdot R_{s 2}+K_{q} \cdot R_{b}
\end{array}\right. \\
\left\{\begin{array}{l}
\left(\frac{\Delta e_{q}}{u_{t}}\right)_{1}=\frac{(c \rho)_{s}}{K_{q}} \cdot h_{1}+\frac{P_{b}}{K_{q}}=\frac{P_{s 1}}{K_{q}}+\frac{P_{b}}{K_{q}} \\
\left(\frac{\Delta e_{q}}{u_{t}}\right)_{2}=\frac{(c \rho)_{s}}{K_{q}} \cdot h_{2}+\frac{P_{b}}{K_{q}}=\frac{P_{s 2}}{K_{q}}+\frac{P_{b}}{K_{q}}
\end{array}\right.
\end{gathered}
$$

As a result of solution of each of these systems of equations with respect to $K_{q}$ we have:

$$
\begin{gathered}
K_{q}=\lambda_{s} \cdot \frac{\left(\frac{\Delta t}{\bar{e}_{q}}\right)_{1}-\left(\frac{\Delta t}{\bar{e}_{q}}\right)_{2}}{h_{1}-h_{2}}=\frac{\left(\frac{\Delta t}{\bar{e}_{q}}\right)_{1}-\left(\frac{\Delta t}{\bar{e}_{q}}\right)_{2}}{R_{s 1}-R_{s 2}}, \\
K_{q}=(c \rho)_{s} \cdot \frac{h_{1}-h_{2}}{\left(\frac{\Delta e_{q}}{u_{t}}\right)_{1}-\left(\frac{\Delta e_{q}}{u_{t}}\right)_{2}}=\frac{P_{s 1}-P_{s 2}}{\left(\frac{\Delta e_{q}}{u_{t}}\right)_{1}-\left(\frac{\Delta e_{q}}{u_{t}}\right)_{2}} .
\end{gathered}
$$

In this case, the formulas for calculating of ballast thermal and volumetric resistances of the TPC device have the following form: 


$$
\begin{aligned}
R_{b} & =\left(\frac{\Delta t}{\bar{e}_{q}}\right)_{1} \cdot K_{q}^{-1}-R_{s 1}=\left(\frac{\Delta t}{\bar{e}_{q}}\right)_{2} \cdot K_{q}^{-1}-R_{s 2}, \\
P_{b} & =K_{q} \cdot\left(\frac{\Delta e_{q}}{u_{t}}\right)_{1}-P_{s 1}=K_{q} \cdot\left(\frac{\Delta e_{q}}{u_{t}}\right)_{2}-P_{s 2} .
\end{aligned}
$$

From the results of the analysis of the systems of equations (12) and (13) it follows that in the case of experiments with samples of the research material, which differ in thickness $h_{1} \neq h_{2}$, that is, the thermal $R_{x 1} \neq R_{x 2}$ and volumetric $P_{x 1} \neq P_{x 2}$ resistances, there is no need to determine the ballast resistances of the device. A similar effect will also appear in the case of a two-cell TPC device (Figure 3), in which two pairs of HFC 3 and two pairs of TC 4 are mounted, respectively, to measure the temperature and the heat flow density on the surfaces of samples 5, 6 of the research material with a thickness of $h_{1} \neq h_{2}$.

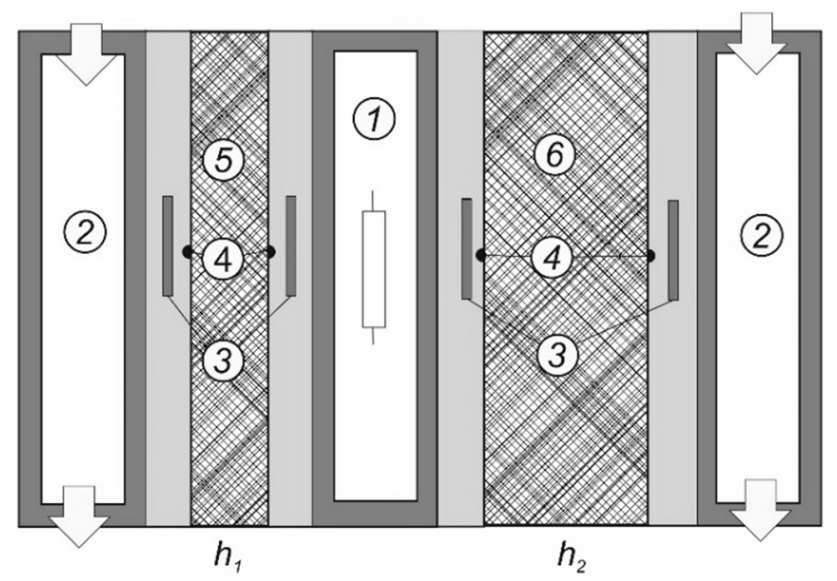

Figure 3. Two-cell device for complex determination of TPC of materials

Since the flow heat exchangers 2 are supplied with heat-transfer material with the same temperature $t_{2}$, which is lower than the temperature $t_{1}$ of the electric heater 1 , and then in the stationary thermal mode, both samples have the same average temperature $\bar{t}=\left(t_{1}+t_{2}\right) / 2$, which includes the results of the calculation of the thermal conductivity of the material according to the formula:

$$
\lambda_{x}=\frac{\bar{q}_{1} \cdot \bar{q}_{2} \cdot\left(h_{1}-h_{2}\right)}{\left(t_{1}-t_{2}\right) \cdot\left(\bar{q}_{1}-\bar{q}_{2}\right)},
$$

where $\bar{q}_{1}$ and $\bar{q}_{2}$ - respectively, are the density of the heat flow that passes through the sample with thickness $h_{1}$ and $h_{2}$.

To determine the volumetric heat capacity of the material, the quantities of heat $Q_{1}$ and $Q_{2}$ are measured, which are accumulated (given) by the samples in the transition mode from one stationary mode to another due to an increase (decrease) in their average temperature by the value $\overline{\delta t}$ and are calculated by the formula: 


$$
(c \rho)_{x}=\frac{Q_{1}-Q_{2}}{\left(h_{x 1}-h_{x 2}\right) \cdot \delta \bar{t}} .
$$

In this case, the operating coefficients of primary HFC and TC, $K_{q}$ and $K_{t}$ of two-cells device, as well as TPC devices of all other modifications, can be determined using special calibration stands of these converters [7-12]. We should note that in the case of the use of special calibration stands, the determination of the operating coefficients of the primary converters of the TPC device occur in conditions that are significantly different from those in which these converters operate during determination of the TPC of materials. Therefore, in some cases, the use of working calibration standards for this purpose may be more appropriate than the use of calibration stands.

At the same time, we note that the existing methods for determining the MC of TPC of device, in particular, using working calibration standards, have certain drawbacks, which include the following:

1. First and foremost it is necessary to take into account that the TPC of any materials, including those that are used as working calibration standards, are determined with certain errors that affect the accuracy of the determination of the TPC of new materials.

2. Contact thermal and volumetric resistances of samples of working calibration standards and prototypes of materials with the working surfaces of the TPC device may differ in value. This is especially true, on the one hand, for the contact resistances of such working calibration standards as, for example, glycerin or quartz glass, and on the other hand prototypes of bulk materials. Since these contact resistances are a component of the ballast resistances of the device, depending on the type of standard material and the research material, it is likely to obtain different values of the ballast thermal and ballast volumetric resistances of the device, and, ultimately, false data on the TPC of the research material.

3. From the results of the analysis of known methods for determining the MC of devices and, in particular, devices for complex determination of TPC of materials, as well as the results of their implementation in practice, it follows that the values of the MC of device are related to each other and in the case of determining one of them with a certain error, the accuracy of determining of other characteristics can be extremely unsatisfactory. To this we add that the change in the value of one of the characteristics of the device in the process of studying the TPC of material can cause a change in the values of its other MC of device, which ultimately leads to the receipt of inaccurate information in relation to the TPC of research material. At the same time, despite the existing shortcomings, the method of determining the MC of devices for complex determination of TPC of materials with the use of working calibration standards in practice is quite common.

On the basis of the above it follows that in order to improve the accuracy of the complex determination of TPC of materials, the MC of the device should be determined by the results of experiments with the research material, and not with the standard material. At the same time, we note that in the case of using the research material as a working calibration standard, the data on the values of the MC of TPC device may in fact become unnecessary.

Graphically, the results of the determination of MC of the device for complex determination of TPC of materials for tests in two samples of standard material of known thermal conductivity $\lambda_{s}$ and the volumetric heat capacity $(c \rho)_{s}$, which differ in the thickness $h_{1} \neq h_{2}$, therefore, thermal $R_{s 1} \neq R_{s 2}$ and volumetric $P_{s 1} \neq P_{s 2}$ resistances shown in Figure $4 a$-f. 
Straight lines $\left(\Delta t / \bar{e}_{q}\right)$ from $R_{s}$ and $\left(\Delta t / \bar{e}_{q}\right)$ from the thickness of the sample of the working calibration standard $h_{s}$ cross the ordinate axis of their graphs (Figure $4 a$ and $b$ ) at point $\mathrm{A}$ at and have angles of inclination to the abscissa axis, respectively, $\varphi=\operatorname{arctq}\left(K_{q}\right)$ and $\psi=\operatorname{arctq}\left(K_{q} / \lambda_{s}\right)$. These lines cross the abscissa axis at $\left|-R_{x}\right|=R_{b}$, that is, at the point where the thermal resistance of the sample of the working calibration standard is equal to the ballast thermal resistance of the TPC device and at $\left|-h_{\lambda}\right|=\lambda_{s} \cdot R_{b}$, where $\left|-h_{\lambda}\right|$ is the thickness of the sample of the working calibration standard at which the thermal resistance of the sample is equal to the ballast thermal resistance of the device $\left|-h_{\lambda}\right| / \lambda_{s}=\left|-R_{s}\right|=R_{b}$.

The lines $\left(\Delta e_{q} / u_{t}\right)$ from $P_{s}$ and $\left(\Delta e_{q} / u_{t}\right)$ from $h_{s}$ cross the ordinate axis of their graphs (Figure $4 d$ and $e$ ) at point $\mathrm{B}$ at $\left(\Delta e_{q} / u_{t}\right)=P_{b} / K_{q}$ and have an angle of inclination to the abscissa axis, respectively, $\gamma=\operatorname{arctq}\left(K_{q}\right)$ and $\theta=\operatorname{arct} q\left(1 / K_{q}\right)$. These lines cross the axis of abscissa at $\left|-P_{x}\right|=P_{b}$ respectively, that is, at the point where the capacitive resistance of the sample working calibration standard is equal to the ballast capacitive resistance of the TPC device and at $\left|-h_{(c \rho)}\right|=P_{b} /(c \rho)_{s}$, where $\left|-h_{(c \rho)}\right|$ is the thickness of the sample working calibration standard at which the capacitive resistance of the sample is equal to the ballast capacitive resistance of the device $\left|-h_{(c \rho)}\right| \cdot(c \rho)_{s}=\left|-P_{x}\right|=P_{b}$.

Such graphs can be built on the results of experiments with any material. At the same time, since the value of the operating coefficient of the HFC of the device does not depend on the TPC of the research material, the ordinates of crossing of the lines $\left(\Delta t / \bar{e}_{q}\right)=f\left(R_{x}\right)$ and $\left(\Delta e_{q} / u_{t}\right)=f\left(P_{x}\right)$ with the axes of the corresponding graphs remain unchanged. The coordinates of the intersection of the lines $\left(\Delta t / \bar{e}_{q}\right)=f(h)$ and $\left(\Delta e_{q} / u_{t}\right)=f(h)$ with the axis of the ordinates of the graphs also remain unchanged when increasing or decreasing the values $\lambda_{x}$ and $(c \rho)_{x}$ of the material. At the same time, when the TPC of the material changes, due to the change in the coordinates of the concurrence of these lines with the abscissa axis, the angles of the lines to the abscissa axis of the graphs change. Therefore, for this kind of dependency $\varphi=f\left(\lambda_{x}\right), \gamma=f(c \rho)_{x}$.

We should note that since the ballast resistances of the device are not a function of the TPC of the material, $R_{b} \neq f(\lambda)_{x}$ and $P_{b} \neq f(c \rho)_{x}$, then with the change of the TPC of the abscissa material $\left|-h_{\lambda}\right|$ and $\left|-h_{(c \rho)}\right|$, they change so that the numerical values of the ratio $\left|-h_{\lambda}\right| / \lambda_{s}=R_{b}$ and the product $\left|-h_{(c \rho)}\right| \cdot(c \rho)_{s}=P_{b}$ remain unchanged.

It follows from the above that for $\lambda_{x}=1$, the angle $\psi$ of the slope of the straight line $\left(\Delta t / \bar{e}_{q}\right)=f(h)$ to the axis of the abscissa (Figure $\left.4 b\right)$ is equal to the angle $\varphi$ of the slope of the straight line $\left(\Delta t / \bar{e}_{q}\right)=f\left(R_{x}\right)$ to $R_{x}$ axis (Figure $\left.4 a\right)$. In this case, the lines $\left(\Delta t / \bar{e}_{q}\right)$ from $h$ and $\left(\Delta t / \bar{e}_{q}\right)$ from $R_{x}$ cross the abscissa axis of their graphs at $\left|-h_{\lambda}\right|=\lambda_{x} \cdot R_{b}=1 \cdot R_{b}=R_{b}$ and $\left|-h_{\lambda}^{\prime}\right| / \lambda=\left|-h_{\lambda}\right| /\left(\lambda_{x} \cdot 1\right)=R_{b}$. Here $\left|-h_{\lambda}^{\prime}\right|$ is the corrected thickness of the ballast layer of thermal resistance at the thermal conductivity of the layer $\lambda_{b}=1$. 
— Processes and Equipment-_
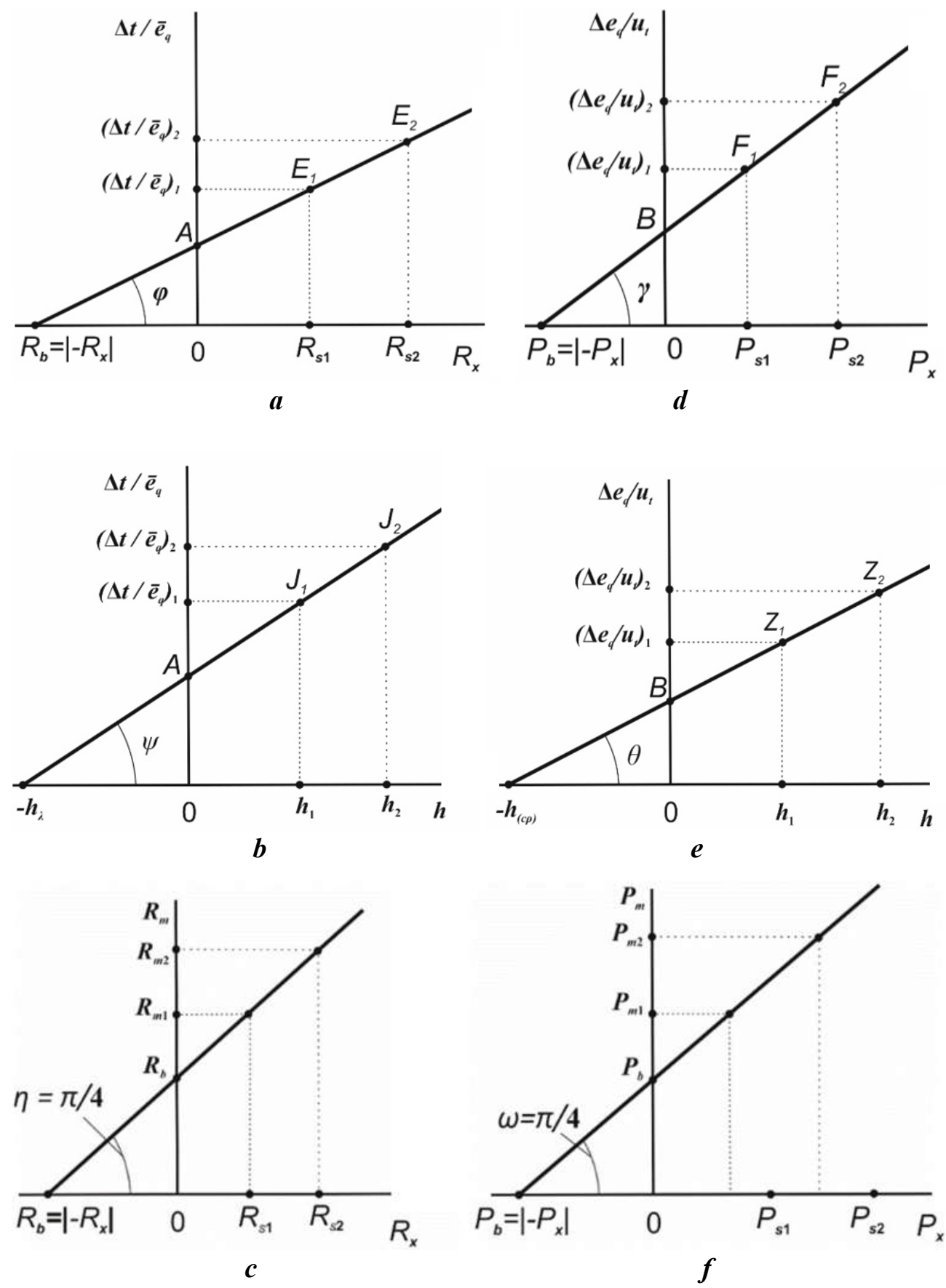

Figure 4. Results of determination of MC of the device of complex determination of TPC of materials during experiments with two samples of standard material 


\section{— Processes and Equipment-}

Similar, at $(c \rho)_{x}=1$, takes place with dependencies $\left(\Delta e_{q} / u_{t}\right)=f(h)$ and $\left(\Delta e_{q} / u_{t}\right)=f\left(P_{x}\right)$ which are shown in Figure $4 e$ and $d$. The angle $\theta$ of inclination of the line $\left(\Delta e_{q} / u_{t}\right)=f(h)$ to the axis $h$ will be equal to the angle $\gamma$ of inclination of the line $\left(\Delta e_{q} / u_{t}\right)=f\left(P_{x}\right)$ to the axis $P_{x}$, while the lines cross the abscissa axis of their graphs at $\left|-h_{(c \rho)}\right|=P_{b} /(c \rho)_{x}=P_{b} / 1=P_{b}$ and $\left|-h_{(c \rho)}^{\prime}\right| \cdot(c \rho)=\left[\left|-h_{(c \rho)}\right| \cdot(c \rho)_{x}\right] / 1=P_{b}$ where $\left|-h_{(c \rho)}^{\prime}\right|$ is the corrected thickness of the capacitive ballast resistance layer at the volumetric heat capacity of the layer $(c \rho)_{b}=1$.

Thus, at $\lambda_{x}=(c \rho)_{x}=1$ the graph $\left(\Delta t / \bar{e}_{q}\right)$ of $h$ is transformered to the view $\left(\Delta t / \bar{e}_{q}\right)$ from $R_{x}$, and $\left(\Delta e_{q} / u_{t}\right)$ of $h$, respectively, to a graph $\left(\Delta e_{q} / u_{t}\right)$ of $P_{x}$. At that the sum of the angles of inclination of the lines $\left(\Delta t / \bar{e}_{q}\right)=f(h)$ and $\left(\Delta e_{q} / u_{t}\right)=f(h)$ to the abscissa axis of the corresponding graphs is $\pi / 2$, that is, for both lines $\left(\Delta t / \bar{e}_{q}\right)=f\left(R_{x}\right)$ and $\left(\Delta e_{q} / u_{t}\right)=f\left(P_{x}\right)$.

Subsequent to the results of determining the MC of device the dependencies $R_{m}$ of $R_{x}$ and $P_{m}$ of $P_{x}$ can be built (Figure $4 c$ and $f$ ). Based on the analysis of equations (8) and (9) it follows that at $\lambda=(c \rho)=1$ these lines intersect the ordinate axis $\left(R_{x}=0\right)$ and the abscissa axis $\left(R_{m}=0\right)$ of their graphs at points with coordinates, respectively:

$$
\begin{gathered}
R_{m}=\Delta t /\left(\bar{e}_{q} \cdot K_{q}\right)=\left(K_{q} \cdot R_{b}\right) / K_{q}=R_{b} \text { and } P_{m}=\left(\Delta e_{q} \cdot K_{q}\right) / u_{t}=\left(P_{b} / K_{q}\right) \cdot K_{q}=P_{b}, \\
\left|-R_{m}\right|=\left|-h_{\lambda}^{\prime}\right| / \lambda=\left|-h_{\lambda}\right| /\left(\lambda_{x} \cdot 1\right)=R_{b} \text { and } \\
\left|-P_{x}\right|=\left|-h_{(c \rho)}^{\prime}\right| \cdot(c \rho)=\left[\left|-h_{(c \rho)}\right| \cdot(c \rho)_{x}\right] / 1=P_{b} .
\end{gathered}
$$

At that the angle of inclination of the lines $R_{m}=f\left(R_{x}\right)$ and $P_{m}=f\left(P_{x}\right)$ to the abscissa axis of the corresponding graph is $\pi / 4$.

It follows from the above that the data on thermal conductivity $\lambda_{\mathrm{s}}$ and the volumetric heat capacity $(c \rho)_{\mathrm{s}}$ of the working calibration standards are used to determine the MC of the TPC device. Thus, according to equations (12)-(17) to determine $R_{b}$ of the device $\lambda_{\mathrm{s}}$ is used, to determine $P_{b}-(c \rho)_{\mathrm{s}}$, and to determine the operating coefficient of HFC of device $\lambda_{\mathrm{s}}$ or $(c \rho)_{\mathrm{s}}$ of working calibration standard is used.

At that the results of the analysis of expressions (12)-(17) show that:

- the system of equations (12) and equations (14) and (16) correspond to the device for determining thermal conductivity of materials;

- the system of equations (13) and equations (15) and (17) correspond to the device for determining the volumetric heat capacity of materials;

- systems of equations (12), (13) and equations (14)-(17) correspond to the device for complex determination of TPC of materials.

At the same time, we note that the method of determining the ballast resistances of the device for complex determination of TPC of materials is a combination of unrelated methods for determining the ballast resistance of the device for determining the thermal conductivity and the device for determining the volumetric heat capacity of materials.

We consider that the method of determination of $\mathrm{MC}$ of the device for complex determination of TPC of materials except $\lambda_{\mathrm{s}}$ and $(c \rho)_{\mathrm{s}}$ first of all shall provide the usage of 
thermal conductivity $a_{s}=\lambda_{s} /(c \rho)_{s}$ and thermal activity $\beta_{s}=\sqrt{\lambda_{s} \cdot(c \rho)_{s}}$ of working calibration standards.

Based on the above, for a combination of the six dependencies shown in Figure $4 a-f$, it is necessary to build their combined graphs.

To build a combined graph of dependencies and $\left(\Delta t / \bar{e}_{q}\right)$ and $\left(\Delta e_{q} / u_{t}\right)$ on $h$ (Figure 5 ), obtained from two experiments with samples of material with thickness $h_{1} \neq h_{2}$, from systems of equations similar to (12), (13) we obtain the coordinates of the intersection of lines $\left(\Delta t / \bar{e}_{q}\right)=f(h)$ and $\left(\Delta e_{q} / u_{t}\right)=f(h)$ with the axes of abscissa and ordinates, as well as the angles of inclination of these lines to the axis of abscissa of their graphs, respectively:

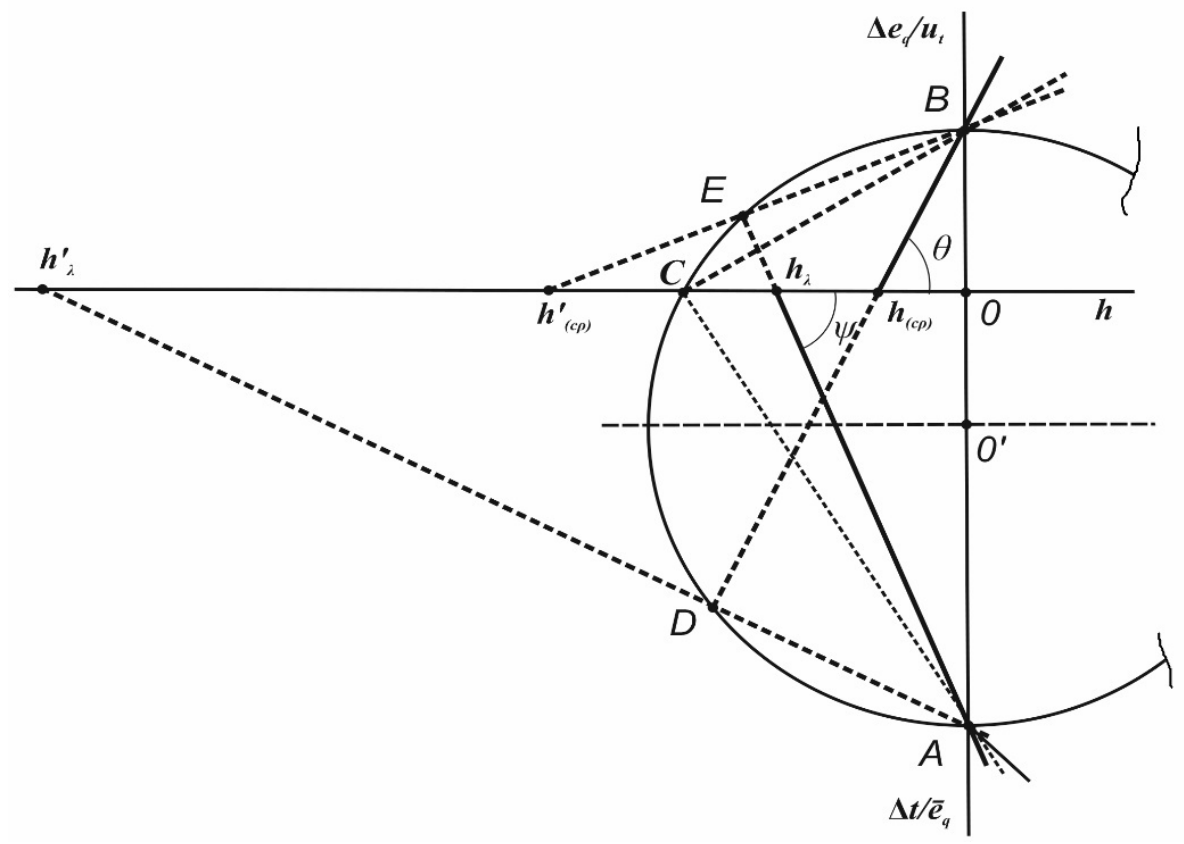

Figure 5. To the construction of a combined dependencies graph

$$
\begin{gathered}
\left(\Delta t / \bar{e}_{q}\right)=f(h) \text { and }\left(\Delta e_{q} / u_{t}\right)=f(h) \\
\left|-\boldsymbol{h}_{\lambda}\right|=\lambda_{x} \cdot \boldsymbol{R}_{\boldsymbol{b}},\left(\Delta t / \bar{e}_{q}\right)=K_{q} \cdot R_{b}=\mathrm{A}, \boldsymbol{\psi}=\operatorname{arctg}\left(\boldsymbol{K}_{q} / \lambda_{x}\right) \\
\quad \text { and } \\
\left|-\boldsymbol{h}_{(\boldsymbol{c} \rho)}\right|=\boldsymbol{P}_{\boldsymbol{x}} /(\boldsymbol{c} \boldsymbol{\rho})_{x},\left(\Delta e_{q} / u_{t}\right)=P_{b} / K_{q}=\mathrm{B}, \boldsymbol{\theta}=\operatorname{arctg}\left[(\boldsymbol{c \rho})_{x} / \boldsymbol{K}_{q}\right] .
\end{gathered}
$$

On the abscissa axis in the selected scale $M_{h}$, we apply a linear scale $h$. To match the scales and values on the abscissa axis $M_{h}$ and the axes of the ordinates $\left(\Delta t / \bar{e}_{q}\right)-M_{R m}$ and $\left(\Delta e_{q} / \Delta u_{t}\right)-M_{P m}$ from the point $-h_{\lambda}$ of the intersection of the line $\left(\Delta t / \bar{e}_{q}\right)=f(h)$ with the abscissa axis, through the third quadrant of the graph, at an angle $\psi=\operatorname{arctg}\left(K_{q} / \lambda_{x}\right)$ and from the point $-h_{(c \rho)}$, the intersection of the line $\left(\Delta e_{q} / u_{t}\right)=f(h)$ with the abscissa axis through the second quadrant of the graph at an angle $\theta=\operatorname{arctg}\left[(c \rho)_{x} / K_{q}\right]$ to the abscissa axis, and draw 
the corresponding lines. In this case, the straight line $\left(\Delta t / \bar{e}_{q}\right)=f(h)$ will cross the ordinate axis at point $\mathrm{A}$, which is located on the "conditionally" negative part of the ordinate axis at a distance of $\mathrm{L}_{0 \mathrm{~A}}$ from the origin of coordinates at $\left(\Delta t / \bar{e}_{q}\right)=K_{q} \cdot R_{b}$, and the straight line $\left(\Delta e_{q} / u_{t}\right)=f(h)-$ at point $\mathrm{B}$, which is in the positive part of the ordinate axis at a distance of $\mathrm{L}_{0 \mathrm{~B}}$ from the origin of the combined chart at $\left(\Delta e_{q} / u_{t}\right)=P_{b} / K_{q}$.

By the values of $h, R_{x}, P_{x}$, the abscissa axis of the graph should be common for two pairs of lines - a pair of lines $\left(\Delta t / \bar{e}_{q}\right)$ from $h$ and $\left(\Delta e_{q} / u_{t}\right)$ from $h$ and a pair of lines $\left(\Delta t / \bar{e}_{q}\right)$ from $R_{x}$ and $\left(\Delta e_{q} / u_{t}\right)$ from $P_{x}$. The lines $\left(\Delta t / \bar{e}_{q}\right)=f(h)$ and $\left(\Delta e_{q} / u_{t}\right)=f(h)$ have an angle of inclination to the abscissa axis of the combined graph $\psi$ and $\theta$ and cross this axis at points, respectively, $\left|-h_{\lambda}\right|=\lambda_{x} \cdot R_{b}$ and $\left|-h_{(c \rho)}\right|=P_{x} /(c \rho)_{x}$.

In contrast to the previous pair, the lines $\left(\Delta t / \bar{e}_{q}\right)=f\left(R_{x}\right)$ and $\left(\Delta e_{q} / u_{t}\right)=f\left(P_{x}\right)$ must have an angle of inclination to the abscissa axis of the combined graph $\varphi=\operatorname{arctq}\left(K_{q}\right)$ and $\gamma=\operatorname{arctq}\left(1 / K_{q}\right)$ and cross the abscissa axis at points which coordinates are numerically equal:

$$
\begin{gathered}
\frac{\left|-h_{\lambda}^{\prime}\right|}{\lambda_{b}}=\frac{\left|-h_{\lambda}\right| / \lambda_{x}}{1}=R_{b}, \\
\left|-h_{(c \rho)}^{\prime}\right| \cdot(c \rho)_{b}=\left[\left|-h_{(c \rho)}\right| \cdot(c \rho)_{x}\right] \cdot 1=P_{b},
\end{gathered}
$$

where $\left|-h_{\lambda}^{\prime}\right|$-effective thickness of ballast layer, which at thermal conductivity of material of this layer $\lambda_{b}=1$ is numerically equal to ballast thermal resistance of TFH device; $\left|-h_{(c \rho)}^{\prime}\right|$ - effective thickness of the ballast layer, which, when the volume heat of the material of this layer $(c \rho)_{b}=1$, is numerically equal to the ballast capacitive resistance of the TFH device.

Therefore, on the abscissa axis of the combined graph, the values are intercepted, which although have the dimensionality $[\mathrm{m}],\left[\mathrm{K} \cdot \mathrm{m}^{2} / \mathrm{W}\right],\left[\mathrm{J} / \mathrm{m}^{2} \cdot \mathrm{K}\right]$, but numerically are equal to the thickness of the material sample. Thus, when intercepted to the abscissa axis of the graph of a uniform scale $h$ (also $R_{x}$, also $P_{x}$ ), at each point of the scale, the thermal and capacitive resistances of the sample of the research material should be numerically equal to each other, $R_{x}=P_{x}$, that is $h / \lambda_{x}=h \cdot(c \rho)_{x}$, where $\lambda_{x} \cdot(c \rho)_{x}=\beta^{2}=1$.

Under these circumstances, for $\lambda_{b}=(c \rho)_{b}=1$, the coordinate of point A, $\left(\Delta e_{t} / \bar{e}_{q}\right)_{\mathrm{A}}=K_{q} \cdot R_{e}$, and the ordinate of point $\mathrm{B}\left(\Delta \tau \cdot \Delta e_{q} / \Delta e_{t}\right)_{\mathrm{B}}=P_{b} / K_{e}$, the combined graph (lengths of segments $L_{0 \mathrm{~A}}$ and $L_{0 \mathrm{~B}}$ ) can be given as follows:

$$
\begin{gathered}
\left(\frac{\Delta e_{t}}{\bar{e}_{q}}\right)_{\mathrm{A}}=K_{e} \cdot \frac{h_{R_{b}}}{\lambda_{b}}=K_{e} \cdot \frac{\left|-h_{\lambda}\right|}{\lambda_{x}}=K_{e} \cdot \frac{\left|-h_{\lambda}\right| / \lambda_{x}}{1}=K_{e} \cdot\left|-h_{\lambda}^{\prime}\right|, \\
\left(\frac{\Delta \tau \cdot \Delta e_{q}}{\Delta e_{t}}\right)_{\mathrm{B}}=\frac{h_{P_{b}} \cdot(c \rho)_{b}}{K_{e}}=\frac{\left|-h_{(c \rho)}\right| \cdot(c \rho)_{x}}{K_{e}}=\frac{\left[\left|-h_{(c \rho)}\right| \cdot(c \rho)_{x}\right] \cdot 1}{K_{e}}=\frac{\left|-h_{(c \rho)}^{\prime}\right|}{K_{e}} .
\end{gathered}
$$


As it follows from the results of the dependencies analysis shown in Figure $4 a-f$, at $\lambda_{\mathrm{x}}=(c \rho)_{\mathrm{x}}=1$ each of the two pairs of lines, $\left(\Delta t / \bar{e}_{q}\right)=f(h)$ and $\left(\Delta t / \bar{e}_{q}\right)=f\left(R_{x}\right)$, and also $\left(\Delta e_{q} / u_{t}\right)=f(h)$ and $\left(\Delta e_{q} / u_{t}\right)=f\left(P_{x}\right)$ in the combined graph should be represented by the corresponding line. In turn, these lines will intersect at an angle $\pi / 2$. Thus, since the lines $\left(\Delta t / \bar{e}_{q}\right)=f\left(R_{x}\right)$ and $\left(\Delta e_{q} / u_{t}\right)=f\left(P_{x}\right)$, as well as $\left(\Delta t / \bar{e}_{q}\right)=f(h)$ and $\left(\Delta e_{q} / u_{t}\right)=f(h)$ , passing through the points $\mathrm{A}$ and $\mathrm{B}$ at $\lambda_{x}=(c \rho)_{x}=1$, i.e. at $a_{\mathrm{x}}=1$ and $\beta_{x}=1$, on the combined graph should intersect at an angle $\pi / 2$, we draw a circle through the points A and B with a diameter:

$$
D=L_{0 \mathrm{~A}}+L_{0 \mathrm{~B}}=\left(\Delta t / \bar{e}_{q}\right)_{0 \mathrm{~A}}+\left(\Delta e_{q} / u_{t}\right)_{0 \mathrm{~B}}
$$

the center of $\mathrm{O}^{\prime}$ of which is on the ordinate axis and generally does not coincide with the origin of the graph.

This circle crosses the abscissa axis of the graph at point $C$, the abscissa of which is the length of the segment $0-C$, can be calculated as geometric mean of lengths of the segments $0-A$ and $0-B$, which form the diameter of the circle. However, given the consistency of the scale of values that are intercepted on the axes of the graph, the abscissa of point $\mathrm{C}$ can be calculated as follows:

$$
h_{C}=\sqrt{L_{0 \mathrm{~A}} \cdot L_{0 \mathrm{~B}}}=\sqrt{\left(\Delta t / \bar{e}_{q}\right)_{0 \mathrm{~A}} \cdot\left(\Delta e / u_{t}\right)_{0 \mathrm{~B}}}=\sqrt{\left(K_{q} \cdot R_{b}\right) \cdot\left(P_{b} / K_{q}\right)}=\sqrt{R_{b} \cdot P_{b}} .
$$

For the convenience of presentation of the material here and further the index " $\mathrm{C}$ " will highlight the values that refer to the point $\mathrm{C}$ on the combined graph.

At Figure 5 the lines $\left(\Delta t / \bar{e}_{q}\right)=f(h)$ and $\left(\Delta e_{q} / u_{t}\right)=f(h)$ intersect the circle in points, respectively, E and D. With the increase of $\lambda_{x}$, the point $\left|-h_{\lambda}\right|=\lambda_{x} \cdot R_{b}$ of intersection of a line $\left(\Delta t / \bar{e}_{q}\right)=f(h)$ with the abscissa axis of the graph moves toward point $\mathrm{C}$ and further in the negative section of the scale. At that the point $\mathrm{E}$ moves in a circle against the clock.

With decreasing of $(c \rho)_{\mathrm{x}}$ the point $\left|-h_{(c \rho)}\right|=P_{x} /(c \rho)_{x}$ of intersection of the line $\left(\Delta e_{q} / u_{t}\right)=f(h)$ with the abscissa axis of the graph moves towards the point $\mathrm{C}$ and further into the negative section of the scale, and the point $\mathrm{D}$ in a circle moves clockwise, that is, to meet the point $\mathrm{E}$.

The position of the points $\mathrm{E}$ and $\mathrm{D}$ on the circle coincide with the point $\mathrm{C}$, when

$$
\left|-h_{C}\right|=\left|-h_{C \lambda}\right|=\left|-h_{\mathrm{C}(c \rho)}\right| .
$$

At that the sum of the angles $\psi_{\mathrm{C}}+\theta_{\mathrm{C}}$ of the inclination of lines $\left(\Delta t / \bar{e}_{q}\right)=f(h)$ and $\left(\Delta e_{q} / u_{t}\right)=f(h)$ to the abscissa axis of the graph is equal to $\pi / 2$ and hence, when $\lambda_{C \mathrm{x}}=(c \rho)_{\mathrm{Cx}}$, $a_{C x}=1$, and -

$$
\frac{h_{C}}{\sqrt{a_{C x}}}=\frac{h_{C}}{1}=\frac{h_{b}}{\sqrt{\lambda_{b} /(c \rho)_{b}}}=\frac{h_{b}}{\sqrt{a_{b}}},
$$

where $h_{R b}, h_{P b}$ and $h_{b}=\sqrt{h_{R b} \cdot h_{P_{b}}}$ - respectively, the thickness of the layer, which causes the ballast thermal and capacitive resistances of the device and the corrected thickness of the 
ballast layer; $\lambda_{b},(c \rho)_{b}$ and $a_{b}$ - respectively, thermal conductivity, volumetric heat capacity and temperature diffusivity of the ballast layer.

In the case where the lines $\left(\Delta t / \bar{e}_{q}\right)=f(h)$ and $\left(\Delta e_{q} / u_{t}\right)=f(h)$ intersect each other in the area $0-\mathrm{C}$, the abscissa axis of the combined graph (Figure 6), for example, at the point $F_{1}$, where $h_{1}=\left|-h_{\lambda 1}\right|=\left|-h_{(c \rho) 1}\right|=R_{x 1}=P_{\mathrm{x} 1}$ or at the point $F_{2}$, де $h_{2}=\left|-h_{\lambda 2}\right|=\left|-h_{(c \rho) 2}\right|=R_{x 2}=P_{\mathrm{x} 2}$, in the the result of the conversion of equations $R_{x 1}=\lambda_{1 x} \cdot R_{b}=P_{\mathrm{x} 1}=P_{b} /(c \rho)_{1 \mathrm{x}}$ and $R_{x 2}=\lambda_{2 x} \cdot R_{b}=P_{\mathrm{x} 2}=P_{b} /(c \rho)_{2 \mathrm{x}}$ we get:

$$
\beta_{1 x}=\beta_{2 x}=\sqrt{P_{b} / R_{b}}=1
$$

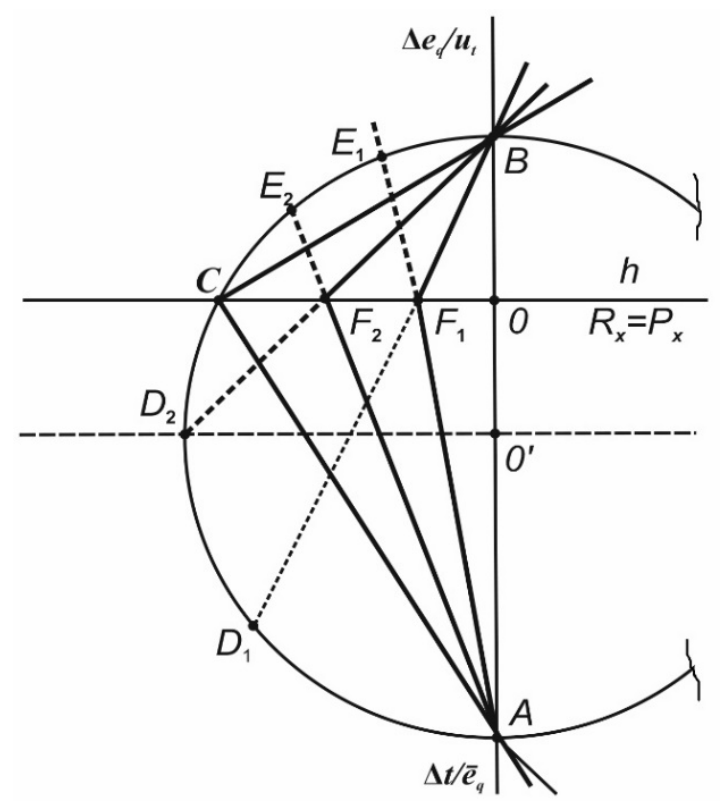

Figure 6. On the analysis of the combined dependencies graph

$$
\left(\Delta t / \bar{e}_{q}\right)=f(h) \text { and }\left(\Delta e_{q} / u_{t}\right)=f(h)
$$

Moving of the point $F$ of the mutual intersection of the lines $\left(\Delta t / \bar{e}_{q}\right)=f(h)$ and $\left(\Delta e_{q} / u_{t}\right)=f(h)$ along the $h$ axis of the graph towards the point $\mathrm{C}$ (Figure 6), is accompanied by an increase in $\lambda_{x}$ and a decrease in $(c \rho)_{x}$, hence an increase in the $a_{x}$ of the research material. The value of the thermal activity of the material does not change, $\beta_{x}=1=$ const. As in the previous case (Figure 5), the change in thermal conductivity, volumetric heat capacity and temperature diffusivity of the research material is accompanied by the movement in a circle of points $\mathrm{E}$ and $\mathrm{D}$ in the direction of the abscissa axis of the graph, that is, the point $C$.

The nature of the dependence of the coordinates of the cross-section of lines $\left(\Delta t / \bar{e}_{q}\right)=f(h)$ and $\left(\Delta e_{q} / u_{t}\right)=f(h)$ with the abscissa axis of the combined graph on the thermal conductivity and volumetric heat capacity of the research material is shown in Figure 7. 
Unlike intersections of lines $\left(\Delta t / \bar{e}_{q}\right)=f(h)$ and $\left(\Delta e_{q} / u_{t}\right)=f(h)$ on the abscissa axis at points $F_{1}$ and $F_{2}$, where $\beta_{1 x}=\beta_{2 x}=\sqrt{P_{b} / R_{b}}=1$, the sum of the angles of inclination of lines to the abscissa axis is not equal to $\pi / 2, a_{1 x} \neq a_{2 x} \neq 1, \lambda_{1 x} \neq(c \rho)_{1 x} \neq 1, \lambda_{2 x} \neq(c \rho)_{2 x} \neq 1$, in the case of intersection of lines at point $\mathrm{C}$, the sum of angles of inclination of lines to the abscissa axis is $\pi / 2$ and $\lambda_{C x}=(c \rho)_{C x}=a_{C x}=\beta_{C x}=\sqrt{P_{b} / R_{b}}=1$ and $R_{b}=P_{b}=\sqrt{R_{b} \cdot P_{b}}$.

The procedure of plotting the combined dependencies graph of $\left(\Delta t / \bar{e}_{q}\right)=f(h)$, $\left(\Delta t / \bar{e}_{q}\right)=f\left(R_{x}\right),(\Delta t / \bar{q})=f\left(R_{x}\right), R_{m}=R_{x}+R_{b},\left(\Delta e_{q} / u_{t}\right)=f(h),\left(\Delta e_{q} / u_{t}\right)=f\left(P_{x}\right)$, $\left(\Delta q / u_{t}\right)=f\left(P_{x}\right)$ and $P_{m}=P_{x}+P_{b}$, based on the results of two experiments with samples of material with thickness $h_{1} \neq h_{2}$ (Figure 8) will be shown on the following example.

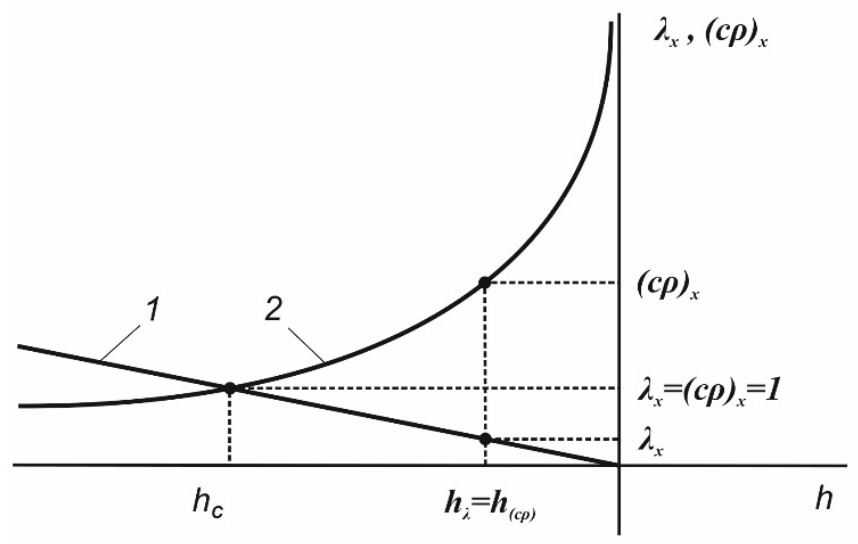

Figure 7. Dependence of thermal conductivity 1 and volumetric heat capacity of 2 experimental material on the coordinate of the intersection of straight lines $\left(\Delta e_{t} / \bar{e}_{q}\right)=f(h)$ and $\left(\Delta \tau \cdot \Delta e_{q} / \Delta e_{t}\right)=f(h)$ with the axis of the abscissus of the combined graph

Based on the results of measurement of the signals $e_{1}, e_{2}$, difference in signals $\Delta e^{=} e_{1}-$ $e_{2}$ and the average signal $\bar{e}_{q}=\left(e_{1}+e_{2}\right) / 2$ of HFC device, temperature $t_{1}, t_{2}$ and the temperature difference $\Delta t=t_{1}-t_{2}$ on the working surfaces of the samples, and the average temperature $\bar{t}=\left(t_{1}+t_{2}\right) / 2$ of samples and the speed of its change $u_{t}=\overline{\delta t} / \Delta \tau$ at heating (cooling) of the samples of research material with a thickness $h_{1} \neq h_{2}$ in quasi-stationary mode: 1. We get the equation of a line $\left(\Delta t / \bar{e}_{q}\right)=\left(K_{q} / \lambda_{x}\right) \cdot h+K_{q} \cdot R_{b}$, that passes through the points $J_{\mathrm{I}}$ and $J_{\mathrm{II}}$, with coordinates, respectively, $\left[h_{1} ;\left(\Delta t / \bar{e}_{q}\right)_{1}\right]$ and $\left[h_{2} ;\left(\Delta t / \bar{e}_{q}\right)_{2}\right]$, and a line $\left(\Delta e_{q} / u_{t}\right)=\left((c \rho)_{x} / K_{q}\right) \cdot h+P_{b} / K_{q}$, that passes through the points $Z_{\mathrm{I}}$ and $Z_{\mathrm{II}}$ with coordinates, respectively, $\left[h_{1} ;\left(\Delta e_{q} / u_{t}\right)_{1}\right]$ and $\left[h_{2} ;\left(\Delta e_{q} / u_{t}\right)_{2}\right]$ that intersect the abscissa and the ordinate axes of their graphs (Figure $4, b$ and $e$ ) at $\left|-h_{\lambda}\right|=\lambda_{\mathrm{x}} \cdot R_{b}$ and point $\mathrm{A}=K_{q} \cdot R_{b}$, and $\left|-h_{(c \rho)}\right|=P_{b} /(c \rho)_{\mathrm{x}}$ and point $\mathrm{B}=P_{b} / K_{q}$. The angles of the lines 1 and 2 relative to the 


\section{- Processes and Equipment-}

abscissa axis of the graph are, respectively: $\psi=\operatorname{arctq}\left(K_{q} / \lambda_{x}\right)$ and $\theta=\operatorname{arctq}\left[(c \rho)_{x} / K_{q}\right]$.

2. By the method of plotting of the combined chart in Figure 5, we build in Figure 8 a combined graph of the line 1 , which is described by the equation $\left(\Delta t / \bar{e}_{q}\right)=\left(K_{q} / \lambda_{x}\right) \cdot h+K_{q} \cdot R_{b}$ and the line 2 , which is described by the equation $\left(\Delta e_{q} / u_{t}\right)=\left[(c \rho)_{x} / K_{q}\right] \cdot h+P_{b} / K_{q}$ and calculate the coordinates of the intersection of the graph circle with the abscissa axis, that is, the values of the corrected ballast thermal and capacitive resistances of the TPC device -

3. Through the points $\mathrm{C}$ and $\mathrm{A}$, the coordinates of which are $\left[-R_{x} ; 0\right]$ and $\left[0 ;\left(K_{q} \cdot R_{b}\right)\right]$, as well as the points $\mathrm{C}$ and $\mathrm{B}$, the coordinates of which are $\left[-P_{x} ; 0\right]$ and $\left[0 ;\left(P_{x} / K_{q}\right)\right]$ we draw the line 3, which is described by the equation $\left(\Delta t / \bar{e}_{q}\right)=K_{q} \cdot R_{x}+K_{q} \cdot R_{b}$ and the line 4 , which is described by the equation $\left(\Delta e_{q} / u_{t}\right)=P_{x} / K_{q}+P_{b} / K_{q}$. At that the tangents of the angles $\varphi$ and $\gamma$, the slope of the lines 3 and 4 relatively to the abscissa axis of the combined graph are, respectively: $\operatorname{tq} \varphi=\left(K_{q} \cdot R_{b}\right) / R_{b}=K_{q} \quad$ and $\operatorname{tq} \gamma=\left(P_{b} / K_{q}\right) / P_{b}=1 / K_{q}$.

$$
R_{b}=P_{b}=\sqrt{\left(K_{q} \cdot R_{b}\right) \cdot\left(P_{b} / K_{q}\right)}=\sqrt{R_{b} \cdot P_{b}} .
$$

4. With the center at the origin of coordinates of the combined graph, we draw the circle with radius $\left|-h_{C}\right|=\left|-R_{x}\right|=\left|-P_{x}\right|=R_{b}=P_{b}$ which crosses the abscissa axis of the graph at point $\mathrm{C}$, and the ordinates axis at points $\mathrm{A}^{\prime}$ and $\mathrm{B}^{\prime}$, the ordinate of which is, respectively: $\left(\Delta t / \bar{e}_{q} \cdot K_{q}\right)=R_{m}=R_{b}$ and $\left(\Delta e_{q} \cdot K_{q} / u_{t}\right)=P_{m}=P_{b}$. In this case, the line 5 , that goes through the points $C$ and $A^{\prime}$ is described by the equation $R_{m}=R_{x}+R_{b}$, and the line 6 , which goes through the points $\mathrm{C}$ and $\mathrm{B}^{\prime}-$ by the equation $P_{m}=P_{x}+P_{b}$. On the combined chart (Figure 9), as well as in Figure 4, $c$ and $f$, the angle of inclination of the lines $5-R_{m}=f\left(R_{\mathrm{x}}\right)$ and $6-P_{m}=f\left(P_{\mathrm{x}}\right)$ to the abscissa axis of the graph is $\pi / 4$.

We should also note that when heating (cooling) material samples in a quasi-stationary thermal mode in a two-cell device (Figure 3), the equation of lines 1-6 can be obtained, and a combined graph similar to the one shown in Figure 8, can be built, for any average temperature of sample of the research material. This allows us to study the dependence of TPC of materials on temperature, taking into account the possible changes in the values of the $\mathrm{MC}$ of device on temperature.

Numerical values of the coefficients of the equations describing the lines $1-6$, obtained from the results of values measuring of HFC and TC of TPC device in the course of experiments from the study of TPC of material, as well as the results of calculations of TPC of this material and $\mathrm{MC}$ of device obtained from the equations describing the lines shown in Figure 8 , are essentially corrected to $a_{x}=\beta_{\mathrm{x}}=1$ and, consequently to $\lambda_{\mathrm{x}}=(c \rho)_{\mathrm{x}}=1$ of the research material. In principle, the MC of the device and the TPC of the research material can be calculated from any of the pairs of equations composed of those describing the line 1,3 , or 5 and the line 2,4 or 6 . 


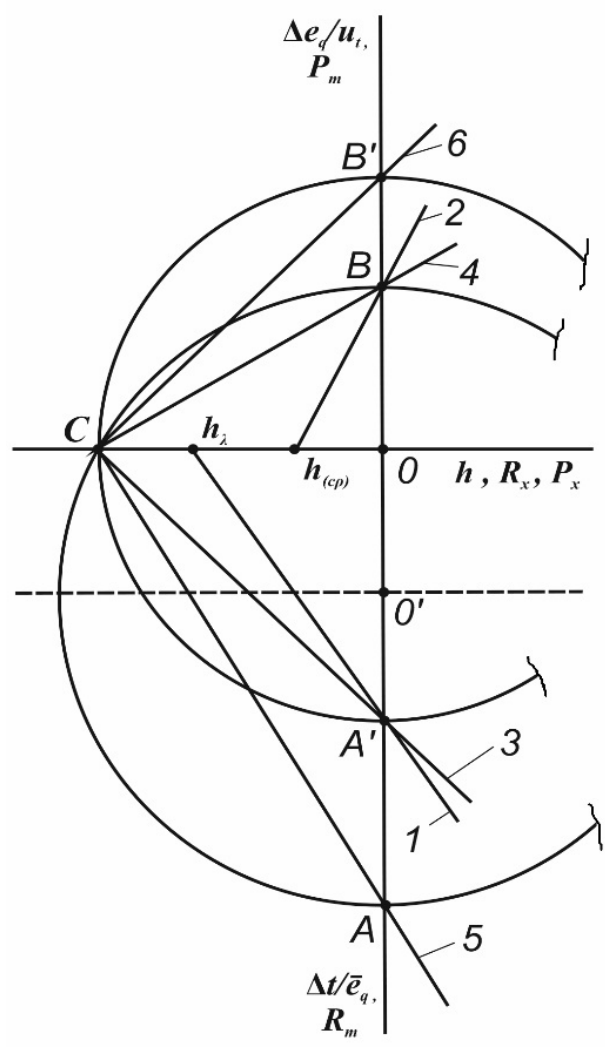

Figure 8. Combined dependencies graph:

$1-\left(\Delta t / \bar{e}_{q}\right)=f(h)$, $2-\left(\Delta e_{q} / u_{t}\right)=f(h)$, 3- $\left(\Delta t / \bar{e}_{q}\right)=f\left(R_{x}\right)$, $4-(\Delta t / \bar{q})=f\left(R_{x}\right)$, $5-R_{m}=R_{x}+R_{b}$, 6- $P_{m}=P_{x}+P_{b}$.

Let's show the calculation procedure of the MC of device and TPC of the research material using the equations that describe the pair of lines: $1-\left(\Delta t / \bar{e}_{q}\right)=f(h)$ and $\left(\Delta e_{q} / u_{t}\right)=f(h), 2-\left(\Delta t / \bar{e}_{q}\right)=f\left(R_{x}\right)$ and $(\Delta t / \bar{q})=f\left(R_{x}\right), 3-R_{m}=R_{x}+R_{b}$ and $P_{m}=P_{x}+P_{b}$.

1. From equations $\left(\Delta t / \bar{e}_{q}\right)=\left(K_{q} / \lambda_{x}\right) \cdot h+K_{q} \cdot R_{b} \quad$ and $\left(\Delta e_{q} / u_{t}\right)=\left[(c \rho)_{x} / K_{q}\right] \cdot h+P_{b} / K_{q}$ we obtain the absissas of lines intersection with the axis of the combined graph $\left|-h_{\lambda}\right|=\lambda_{\mathrm{x}} \cdot R_{b}$ and $\left|h_{(c \rho)}\right|=P_{b} /(c \rho)_{\mathrm{x}}$, then by equation (24) we calculate the value of the corrected thermal and capacitive resistances of the device. Also we determine the value of the operating coefficient of HFC of device, according to the equation -

$$
K_{q}=\sqrt{\frac{K_{q} \cdot R_{b}}{P_{b} / K_{q}} .}
$$

The easiest way to determine the TPC of research material using lines 1 and 2 of the combined graph can be calculated as follows:

$$
\lambda_{x}=\frac{\lambda_{x} \cdot R_{b}}{R_{b}}=\frac{\left|-h_{\lambda}\right|}{\left|-h_{C}\right|},
$$




$$
\begin{gathered}
(c \rho)_{x}=\frac{P_{b}}{P_{b} /(c \rho)_{x}}=\frac{\left|-h_{C}\right|}{\left|-h_{(c \rho)}\right|}, \\
a_{x}=\frac{\lambda_{x}}{(c \rho)_{x}}=\frac{\left|-h_{\lambda}\right| \cdot\left|-h_{(c \rho)}\right|}{\left|-h_{C}\right|^{2}} \\
\beta_{x}=\sqrt{\lambda_{x} \cdot(c \rho)_{x}}=\sqrt{\frac{\left|-h_{\lambda}\right|}{\left|-h_{(c \rho)}\right|}}
\end{gathered}
$$

It was noted above that the values of the coefficients of the equations describing the lines shown in Figure 8, including line $1-\left(\Delta t / \bar{e}_{q}\right)=\left(K_{q} / \lambda_{x}\right) \cdot h+K_{q} \cdot R_{b}$ and line $2-$ $\left(\Delta e_{q} / u_{t}\right)=\left[(c \rho)_{x} / K_{q}\right] \cdot h+P_{b} / K_{q}$, are corrected to $a_{x}=\beta_{\mathrm{x}}=1$. To substantiate this statement, we assume that the line $\left(\Delta t / \bar{e}_{q}\right)=f(h)$ and line $\left(\Delta e_{q} / u_{t}\right)=f(h)$, built on a combined graph (Figure 5) according to the results of experiments with samples of two materials that have temperature diffusivity $a_{\lambda}=a_{(c \rho)}=1$.

Under such conditions, we will show the procedure for determining the thermal conductivity $\lambda_{\mathrm{x}}$ of the material, which in Figure 5 corresponds to the line crossing the ordinate axis of the combined graph at point $\mathrm{A}$, the abscissa axis at $\left|-h_{\lambda}\right|=\lambda_{\mathrm{x}} \cdot R_{b}$ and the circle of the graph at point $\mathrm{E}$, and the procedure of determining the volumetric heat capacity $(c \rho)_{\mathrm{x}}$ of the second material, which corresponds to the line crossing the ordinate axis of the combined graph at point $\mathrm{B}$, the abscissa axis at $\left|-h_{(c \rho)}\right|=P_{b} /(c \rho)_{x}$ and the circle at point $\mathrm{D}$, graphically.

To do this, we draw a line $\left(\Delta e_{q} / u_{t}\right)^{\prime}=f(h)$ through the points $\mathrm{B}$ and $\mathrm{E}$, which is shown on the combined graph as a dashed line, until crossing the abscissa axis of the graph. Given that $a_{\lambda}=1$ abscissa of intersection of this line with the axis of the graph can be written as follows:

$$
\left|-h_{(c \rho)}^{\prime}\right|=\frac{P_{b}^{\prime}}{(c \rho)_{\lambda}}=\frac{P_{b}^{\prime}}{\lambda_{x}}=\frac{\lambda_{x} \cdot\left|-h_{(c \rho)}^{\prime}\right|}{\lambda_{x}} .
$$

Through the points $\mathrm{A}$ and $\mathrm{D}$ we draw a line $\left(\Delta t / \bar{e}_{q}\right)^{\prime}=f(h)$, which is shown on the combined graph as a dashed line, until the intersection with the abscissa axis of the graph. Since $a_{(\mathrm{cp})}=1$, the abscissa of the intersection of this line with the axis of the graph will be:

$$
\left|-h_{\lambda}^{\prime}\right|=\lambda_{(c \rho)} \cdot R_{b}^{\prime}=(c \rho)_{x} \cdot R_{b}^{\prime}=(c \rho)_{x} \cdot\left|-h_{\lambda}^{\prime}\right| /(c \rho)_{x} .
$$

Taking into account the equations (30) and (31) and in accordance with (24), the equation for calculating the ballast thermal and capacitive resistances of the device, using which we carry out the determination of $\lambda_{\mathrm{x}}$ and $(c \rho)_{\mathrm{x}}$ of materials, we write:

$$
R_{b}=\sqrt{R_{b} \cdot P_{b}^{\prime}}=h_{C} \text { and } P_{b}=\sqrt{P_{b} \cdot R_{b}^{\prime}}=h_{C} .
$$

As a result of the transformation of these equations: 


$$
\begin{aligned}
& \text { Processes and Equipment- } \\
& \frac{R_{b}}{\sqrt{R_{b} \cdot P_{b}^{\prime}}}=\frac{\sqrt{R_{b}}}{\sqrt{P_{b}^{\prime}}}=\sqrt{\frac{\left|-h_{\lambda}\right|}{\left|-h_{(c \rho)}^{\prime}\right| \cdot \lambda_{x}^{2}}}=\frac{\left|-h_{\lambda}\right|}{\left|-h_{C}\right|} \cdot \lambda_{x}^{-1}=1, \\
& \frac{P_{b}}{\sqrt{P_{b} \cdot R_{b}^{\prime}}}=\frac{\sqrt{P_{b}}}{\sqrt{R_{b}^{\prime}}}=\sqrt{\frac{\left|-h_{(c \rho)}\right| \cdot(c \rho)_{x}^{2}}{\left|-h_{\lambda}^{\prime}\right|}}=\frac{\left|-h_{(c \rho)}\right|}{\left|-h_{C}\right|} \cdot(c \rho)_{x}=1,
\end{aligned}
$$

we obtain equations for calculation of $\lambda_{\mathrm{x}}$ and $(c \rho)_{\mathrm{x}}$ similar to (26) and (27).

2. The procedure for determining the $\mathrm{MC}$ of device using the equation $\left(\Delta t / \bar{e}_{q}\right)=K_{q} \cdot R_{x}+K_{q} \cdot R_{b}$, that describes the line 3 and the equation $\left(\Delta e_{q} / u_{t}\right)=P_{x} / K_{q}+P_{b} / K_{q}$, that describes the line 4 , is fundamentally the same as in the case of using the equations of lines $\left(\Delta t / \bar{e}_{q}\right)=f(h)$ and $\left(\Delta e_{q} / u_{t}\right)=f(h)$. At the same time, to obtain reliable results of the study of the temperature dependencies of the TPC of material, it is necessary to have information on the temperature dependencies of the $\mathrm{MC}$ of device. In this regard, the value of the operating coefficient of HFC $K_{q(\bar{t})}$, as well as ballast resistances $R_{b(\bar{t})}$ and $P_{b(\bar{t})}$ of the device should be calculated according to the equations (24) and (25) at the average temperature $\bar{t}=\left(t_{2}+t_{2}\right) / 2$ of the test sample, which includes the results of the calculation of the TPC of material.

Further, with the use of complexes $\left(\Delta t / \bar{e}_{q}\right)_{\overline{(t)}}$ and $\left(\Delta e_{q} / u_{t}\right)_{(\bar{t})}$, the values of which are determined by the results of measurement of primary HFC and TC of device at the average temperature of the sample $\bar{t}$, we calculate the TPC of the material by the formulas:

$$
\begin{aligned}
& \lambda_{x(\bar{t})}=\frac{K_{q(\bar{t})} \cdot h}{\left(\Delta t / \bar{e}_{q}\right)_{(\bar{t})}-\left(K_{q} \cdot R_{b}\right)_{(\bar{t})}} \\
& (c \rho)_{x(\bar{t})}=\frac{\left(\frac{\Delta e_{q}}{u_{t}}\right)_{(\bar{t})}-\left(\frac{P_{b}}{K_{q}}\right)_{(\bar{t})}}{K_{q(\bar{t})} \cdot h} \\
& a_{x(\bar{t})}=\frac{\lambda_{x(\bar{t})}}{(c \rho)_{x(\bar{t})}}=\left(K_{q(\bar{t})} \cdot h\right)^{2} \cdot\left[\left(\frac{\Delta t}{\bar{e}_{q}}\right)_{\overline{(\bar{t})}}-\left(K_{q} \cdot R_{b}\right)_{\overline{(\bar{t})}}\right]^{-1} \cdot\left[\left(\frac{\Delta e_{q}}{u_{t}}\right)_{\overline{(\bar{t})}}-\left(\frac{P_{b}}{K_{q}}\right)_{\overline{(\bar{t}})}\right]^{-1}, \\
& \beta_{x(\bar{t})}=\sqrt{\lambda_{x(\bar{t})} \cdot(c \rho)_{x(\bar{t})}}=\frac{\left(\frac{\Delta e_{q}}{u_{t}}\right)_{\overline{(\bar{t}})}-\left(\frac{P_{b}}{K_{q}}\right)_{(\bar{t})}}{\left(\frac{\Delta t}{\overline{e_{q}}}\right)_{(\bar{t})}-\left(K_{q} \cdot R_{b}\right)_{\overline{(\bar{t})}}} .
\end{aligned}
$$


3. The procedure for calculating the TPC of material using the equations $R_{m}=R_{x}+R_{b}$, and $P_{m}=P_{x}+P_{b}$, and lines 5 and 6 , differs from the two described above, in no need to calculate the MC of TPC of device before calculating the TPC of the material by formulas:

$$
\begin{gathered}
\lambda_{x(\bar{t})}=\frac{h}{(\Delta t / \bar{q})_{(\bar{t})}-R_{b(\bar{t})}}, \\
(c \rho)_{x(\bar{t})}=\frac{\left(\Delta q / u_{t}\right)_{(\bar{t})}-P_{b(\bar{t})}}{h}, \\
a_{x(\bar{t})}=\frac{\lambda_{x(\bar{t})}}{(c \rho)_{x(\bar{t})}}=h \cdot\left(\frac{\Delta t}{\bar{q}}-R_{b}\right)_{(\bar{t})}^{-1} \cdot\left(\frac{\Delta q}{u_{t}}-P_{b}\right)_{(\bar{t})}^{-1} \\
\beta_{x(\bar{t})}=\sqrt{\lambda_{x(\bar{t})} \cdot(c \rho)_{x(\bar{t})}}=\frac{\left(\Delta q / u_{t}\right)_{(\bar{t})}-P_{b(\bar{t})}}{(\Delta t / \bar{q})_{(\bar{t})}-R_{b(\bar{t})}}
\end{gathered}
$$

\section{Conclusion}

Simultaneous obtaining information on the values of the thermophysical characteristics of the material and the metrological characteristics of the device can improve the accuracy of the study thanks:

- The exclusion of the use of the results of experiments with samples of reference materials for the determination of the metrological characteristics of the device, and, consequently, TFR of the research materials;

- The possibility to take into account the change of the metrological characteristics of the device from the temperature, as well as the resistance of the system "devicesample" during the expriment with the test material.

\section{References}

1. Incropera F.P., DeWitt D.P., Bergman T.L., and Lavine A.S. (2007), Fundamentalsof Heatand Mass Transfer, JohnWiley\&Sons.

2. Eckert E. R. G., and Drake R. M. (1972), Analysis of Heat and Mass Transfer, McGrawHill, New York.

3. Touloukian Y. S., and Ho C. Y., Eds. (1972), Thermophysical Properties of Matter, Vol. 1, Thermal Conductivity of Metallic Solids; Vol. 2, Thermal Conductivity of Nonmetallic Solids; Vol. 4, Specific Heat of Metallic Solids; Vol. 5, Specific Heat of Nonmetallic Solids; Vol. 7, Thermal Radiative Properties of Metallic Solids; Vol. 8, Thermal Radiative Properties of Nonmetallic Solids; Vol. 9, Thermal Radiative Properties of Coatings, Plenum Press, New York.

4. Vargaftik N.B. (1975), Tables of Thermophysical Properties of Liquids and Gases, 2nd ed., Hemisphere Publishing, New York.

5. Lemmon E. W., McLinden M. O., and Huber M. L. (2002), NIST Standard Reference Database 23: Reference Fluid Thermodynamic and Transport Properties-REFPROP, 
Version 7.0 National Institute of Standards and Technology, Standard Reference Data Program, Gaithersburg.

6. Liley P. E. (1984), Tables in SI Units, private communication, School of Mechanical Engineering, Purdue University, West Lafayette, IN.

7. Platunov E.S., Buravoj S.E., Kurepin V.V., Petrov G.S. (1986), Teplofizicheskie izmereniya i pribory, Mashinostroenie, Leningrfd.

8. Hatakeyama T. and Liu Z. (1999), Handbook of Thermal Analysis, John Wiley.

9. Uendlandt U. (1978), Termicheskie metody analiza, Mir, Moscow.

10. Gerashhenko O.A., Gordov A.N. i dr. (1989), Temperaturnye izmereniya Spravochnik, Nauk.dumka, Kiev.

11. Gerashhenko O.A. (1971) Osnovy Teplometrii, Naukova dumka, Kyiv.

12. Grishhenko T.G., Dekusha L.V., Vorobev L.J. (2017), Teplometriya: teoriya, metrologiya, praktika Knsga 1: Metody i sredstva izmereniya teplovogo potoka, Institut texnicheskoj teplofiziki NAN Ukrainy, Kyiv.

13. Burova Z., Vorobjov L., Dekusha L., Dekusha O. (2009), Ustanovka dlya vimiryuvannya koeficienta teploprovidnosti budivelnix materialiv IT-7S, Metrologiya ta priladi: Naukovo-virobnichij zhurnal, 6, pp. 9-15.

14. HFM 436 Lambda-Heat flow meter, Available at: http://www.netzsch-thermalanalysis.

15. Instrument Line / LaserComp, Available at: http: //www. lasercomp. com/product/ default. php. - Title from the screen.

16. Anatyjchuk L.I., Luste O.Ya. (1981) Mikrokalorimetriya, Vishcha shkola, Lviv.

17. Dekusha O.L., Vorobev L.I. (2016) Izmerenie teploemkosti metodom poshagovogo skanirovaniya pri odnovremennom sravnenii s etalonnym obrazcom, Ukriinskij metrologichnij zhurnal., 4, pp. 48-51.

18. Thermocouples (2016), Available at: www.thermocoupleinfo.com/typethermocouple.htm

19. Scientific research / heat and heat transfer measurement, Available at: https://www.hukseflux.com/applications/scientific-research-heat-and-heat-transfermeasurement.

20. Versey N.G., Gore C.J., Halson S.L., Plowman J.S., Dawson B.T. (2011), Validity and reliability of temperature measurement by heat flow thermistors, flexible thermocouple probes and thermistors in a stirred water bath, Physiol Meas, 32(9), pp. 1417-24.

21. Abdelaziz Y. A. (2006), Stability Characteristics of Industrial Platinum Resistance Thermometers, MAPAN Journal of Metrology Society of India, 21(1), pp. 3-7

22. Cvitaš Lj., Hocenski Ž. (2007), Automated Measurement System for Industrial Platinum Resistance Thermometer Manufacturing Industry, Proceedings of the IEEE International Symposium on Industrial Electronics, ISIE, VIGO, Spain, 4-7 June 2007, pp. 1606-1611.

23. Hocenski Ž. Cvitaš Lj. Lasinger Ž. (2008) Comparison of Methods for Nonlinearity Correction of Platinum Resistance Thermometer, SICE Annual Conference 2008 in Chofu, Tokyo, Japan, pp. 3151-3154.

24. Boris B. Hocenski Ž. Cvitaš Lj. (2006), Optimal Approximation Parameters of Temperature Sensor Transfer Characteristic for Implementation in Low Cost Microcontroller Systems, IEEE ISIE Montreal, Canada, pp. 2784-2787.

25. Xi Meng, BiaoYan, Yanna Gao, Jun Wang, Wei Zhang, Enshen Long (2015), Factors affecting the in situ measurement accuracy of the wall heat transfer coefficient using the heat flow meter method,. Energy and Buildings, 86, pp.754-765 